\title{
Systems of particles with interaction and the cluster formation in condensed matter
}

\author{
V.Krasnoholovets; B.Lev ${ }^{\dagger}$ \\ Institute of Physics of the National Academy of Sciences of Ukraine, \\ 46 Nauky Ave., 03028 Kyiv, Ukraine
}

Received December 21, 2000, in final form December 29, 2002

\begin{abstract}
We investigate the behaviour of a system of particles with the different character of interaction. The approach makes it possible to describe systems of interacting particles by statistical methods taking into account a spatial nonhomogeneous distribution of particles, i.e. cluster formation. For these clusters are evaluated: their size, the number of particles in a cluster, and the temperature of phase transition to the cluster state. Three systems are under consideration: electrons on the liquid helium surface, particles interacting by the shielding Coulomb potential, which are found under the effect of an elastic field (e.g. nucleons in a nucleus), and gravitating masses with the Hubble expansion.
\end{abstract}

Key words: statistical mechanics, interparticle interactions, clusters

PACS: $34.10 .+x, 36.90 . f$

\section{Introduction}

Basically, statistical description of many-particle systems concerns homogeneous states. In monograph [1], a general method based on quantum field theory was successfully applied to homogeneous states of condensed media. An approach that took into account spatial nonhomogeneous states of a system of particles was first proposed in paper [2]; the approach was resting on the employment of the methodology of quantum field theory as well. The most general approach to the description of a system of particles, which included the interparticle interaction was offered in paper [3] in which the procedure of discount of the number of states providing for the nonhomogeneous particle distribution was proposed. However, a correct solution of nonlinear equations in the saddle-point is an impossible task for the most part: the general approach does not permit one to obtain analytical solutions for a sys-

*E-mail: krasnoh@iop.kiev.ua

†E-mail: lev@iop.kiev.ua 
tem of particles when the inverse operator of the interaction energy does not exist. In the interim we need to know a series of parameters which characterize possible clusters. The major parameters are the cluster size and the critical temperature of cluster stability. Because of that, one can raise a question about a more simplified approach, which, however, would permit the study of cluster formation in an initially homogeneous system of particles.

In the present work we study a system of interacting particles having regard to the concept proposed in papers $[2,4]$. Such an approach reduces the number of variables, which determine the free energy and introduces a peculiar "combined variable" [5] playing a role of a typical variable that describes the thermodynamics of the system with a spatial nonhomogeneous particle order.

For instance, as an analog of a certain combined variable one can consider the interaction of two waves on the water surface, which interfere in a limited range. However, after the interference, the waves depart and hence in this case the combined variable does not bring into existence any instability of the system studied. On the other hand, the interaction of an incident electromagnetic wave with a polar crystal results in the polariton, a stable formation, which is indeed described by a combined (canonical) variable - a mixture of the electromagnetic field and optical phonon.

Can the combined variable give rise to the change in the homogeneous distribution of interacting particles? We analyze such an option considering concrete examples. Below we take a look at the specific systems with long-range repulsion/attraction and short-range attraction/repulsion. In particular, the approach provides a way of estimating the cluster size, the number of particles in a cluster and the temperature of phase transition to the cluster state. The approach is examined in three examples: i) electrons on the liquid helium surface, ii) particles with the shielding Coulomb interaction, which are found in an applied elastic potential, and iii) gravitating masses with the Hubble expansion.

\section{Statistical mechanics for model systems with interaction. The principle of states selection}

Let us consider a model system of particles with arbitrary kinds of interactions. Nevertheless, the de Broglie thermal wavelength $\lambda$ of a particle is taken to be greater than the mean distant $l$ between particles. However on the other hand, the value of $\lambda$ should be smaller than the mean amplitude of scattering. The type of the statistics introduces significant peculiarities to the interparticle interaction, which, however, can be rendered by the classical methods allowing one to do away with dynamic quantum correlations. In this case the macroscopic state of the system in question may be specified by filling numbers $n_{s}$, where the symbol $s$ describes the state of the system. In other words, particles can occupy only the knots that are still unoccupied. This means that the filling numbers $n_{s}$ can run only two meanings: 1 (the $s$-th knot is occupied) or 0 (the $s$-th knot is not occupied). Besides, the allocation of particles in the lattice invites the type of statistics, which set limits to the behaviour of particles, i.e. the statistics refers particles to either bosons or fermions, see below. 
The Hamiltonian of the system of interacting particles can be written in the form

$$
\mathcal{H}(n)=\sum_{s} E_{s} n_{s}-\frac{1}{2} \sum_{s s^{\prime}} v_{s s^{\prime}} n_{s} n_{s^{\prime}}+\frac{1}{2} \sum_{s s^{\prime}} u_{s s^{\prime}} n_{s} n_{s^{\prime}}
$$

where $E_{s}$ is the additive part of the particle energy in the $s$-th state; $v_{s s^{\prime}}$ and $u_{s s^{\prime}}$ are, respectively, the paired energy of attraction and repulsion between particles in the states $s$ and $s^{\prime}$. Signs before the potentials in expression (1) reflect the proper signs of the attractive and repulsive paired energies and, therefore, both of the functions $v_{s s^{\prime}}$ and $u_{s s^{\prime}}$ are positive.

Such writing of the Hamiltonian corresponds to the model in which particles are found in sites of the three-dimensional lattice. As will be evident from the further consideration, a specific form of the lattice will be unnecessary. However the medium will be supposed isotropic at the transition to the continual approximation.

Note that the Hamiltonian of the model of replacement of solid solutions [6] amounts to the Hamiltonian (1) as well. This means that the proposed approach can be used to describe the behaviour of defects in solids, alloys, liquids, etc. (in these cases one particle will characterize an unfilled place and another particle will characterize a filled one). The reduced solution Hamiltonian can be amounted to a general Ising model, which takes into account an arbitrary interaction allowing for the spatial-nonhomogeneous distribution of particles.

The partition function of the system is

$$
Z=\sum_{\left\{n_{s}\right\}} \exp \left(-\mathcal{H}\left(n_{s}\right) / k_{\mathrm{B}} T\right)
$$

where the summation provides for all possible values $n_{s}$, i.e., all states of the system. Such summation can be formally performed, if one passes to field variables according to [2]. It is known from the theory of Gauss integrals that

$$
\exp \left[\frac{\nu^{2}}{2} \sum_{s s^{\prime}} w_{s s^{\prime}} n_{s} n_{s^{\prime}}\right]=\operatorname{Re} \int_{-\infty}^{\infty} D \chi \exp \left[\nu \sum_{s} n_{s} \chi_{s}-\frac{1}{2} \sum_{s s^{\prime}} w_{s s^{\prime}}^{-1} \chi_{s} \chi_{s^{\prime}}\right]
$$

where $D \chi \equiv \Pi_{s} \sqrt{\operatorname{det}|| W_{s s^{\prime}}||} \sqrt{2 \pi} d \chi_{s}$ implies the functional integration with respect to the field $\chi$ and $\nu^{2}= \pm 1$ in relation to the sign of interaction $(+1$ for attractive interaction and -1 for repulsion). Besides the obligatory condition

$$
\sum_{s^{\prime \prime}} w_{s s^{\prime \prime}}^{-1} w_{s^{\prime \prime} s^{\prime}}=\delta_{s s^{\prime}}
$$

is fulfilled. In particular, condition (4), in principle, could allow one to determine the inverse operator $w_{s s^{\prime}}^{-1}$ (in the case when it exists) which in its turn would permit the construction of the Green function for interacting particles.

Let us introduce the dimensionless energies $\tilde{w}_{s s^{\prime}} \equiv w_{s s^{\prime}} / k_{\mathrm{B}} T$ and $\tilde{E}_{s} \equiv E_{s} / k_{\mathrm{B}} T$. Note that hereinafter the tilde over a symbol means the division of the symbol by $k_{\mathrm{B}} T$. 
So the partition function (2) can be presented in the form

$$
\begin{aligned}
Z=\operatorname{Re} & \int_{-\infty}^{\infty} D \phi \int_{-\infty}^{\infty} D \psi \sum_{\left\{n_{s}\right\}} \exp \left[-\sum_{s} \tilde{E}_{s} n_{s}+\sum_{s}\left(\psi_{s}+\mathrm{i} \phi_{s}\right) n_{s}\right. \\
& \left.-\frac{1}{2} \sum_{s s^{\prime}}\left(\tilde{u}_{s s^{\prime}}^{-1} \phi_{s} \phi_{s^{\prime}}+\tilde{v}_{s s^{\prime}}^{-1} \psi_{s} \psi_{s^{\prime}}\right)\right] .
\end{aligned}
$$

We do not include the dependence on momentum of particles into the functional integrals in the partition function (5); the sum takes into consideration only the spatial distribution of particles and their energy.

Now we can settle the quantity of particles in the system, $\sum_{s} n_{s}=N$. The procedure is an equivalent to the consideration of the canonical ensemble. For this purpose one can use the formula known in the theory of complex variable

$$
\frac{1}{2 \pi \mathrm{i}} \oint \mathrm{d} z z^{N-1-\sum_{s} n_{s}}=1
$$

It makes it possible to introduce the sum of the canonical ensemble

$$
\begin{array}{r}
Z_{N}=\operatorname{Re} \frac{1}{2 \pi \mathrm{i}} \oint \mathrm{d} z \int D \phi \int D \psi \exp \left\{-\frac{1}{2} \sum_{s s^{\prime}}\left(\tilde{u}_{s s^{\prime}}^{-1} \phi_{s} \phi_{s^{\prime}}+\tilde{v}_{s s^{\prime}}^{-1} \psi_{s} \psi_{s^{\prime}}\right)\right. \\
+(N-1) \ln z\} \sum_{\left\{n_{s}\right\}} \exp \left\{\sum_{s} n_{s}\left(\psi_{s}+\mathrm{i} \phi_{s}-\tilde{E}_{s}-\ln z\right)\right\}
\end{array}
$$

Summing over $n_{s}$ (note $\left.\left\{n_{s}\right\}=0,1\right)$ we get

$$
Z=\operatorname{Re} \frac{1}{2 \pi \mathrm{i}} \int D \phi \int D \psi \oint \mathrm{d} z \mathrm{e}^{S(\phi, \psi, z)}
$$

where

$$
\begin{aligned}
S= & \sum_{s}\left\{-\frac{1}{2} \sum_{s^{\prime}}\left(\tilde{u}_{s s^{\prime}}^{-1} \phi_{s} \phi_{s^{\prime}}+\tilde{v}_{s s^{\prime}}^{-1} \psi_{s} \psi_{s^{\prime}}\right)+\eta \ln \left|1+\frac{\eta}{z} \mathrm{e}^{-\tilde{E}_{s}} \mathrm{e}^{\psi_{s}} \cos \phi_{s}\right|\right\} \\
& +(N-1) \ln z .
\end{aligned}
$$

Here, the function $\eta$ is equal to \pm 1 (Fermi or Bose statistics), see e.g. [7-9]. This provides the means determining the necessary procedure of the states selection that introduces the most essential contribution to the partition function and defines the free energy of the system. Let us set $z=\xi+\mathrm{i} \zeta$ and consider the action $S$ on the transit path passing through the saddle-point with a fixed imaginable variable $\operatorname{Im} z=\zeta_{0}$. In this case, it stands to reason that the action $S$, similarly to quantum field theory, is the variational functional that depends on three variables: the fields $\phi_{s}$ and $\psi_{s}$, and the fugacity $\xi$ (here $\xi=\mathrm{e}^{-\mu / k_{\mathrm{B}} T}$ where $\mu$ is the chemical potential). The extremum of the functional should be realized at solutions of the equations 
$\delta S / \delta \phi_{s}=0, \delta S / \delta \psi_{s}=0$, and $\delta S / \delta \xi=0$. These equations appear as follows:

$$
\begin{gathered}
\sum_{s^{\prime}} \tilde{u}_{s s^{\prime}}^{-1} \phi_{s^{\prime}}=-\frac{\mathrm{e}^{-\tilde{E}_{s}} \mathrm{e}^{\psi_{s}} \sin \phi_{s}}{\xi+\eta \mathrm{e}^{-\tilde{E}} \mathrm{e}^{\psi_{s}} \cos \phi_{s}}, \\
\sum_{s^{\prime}} \tilde{v}_{s s^{\prime}}^{-1} \psi_{s^{\prime}}=\frac{\mathrm{e}^{-\tilde{E}_{s}} \mathrm{e}^{\psi_{s}} \cos \phi_{s}}{\xi+\eta \mathrm{e}^{-\tilde{E}} \mathrm{e}_{s} \cos \phi_{s}}, \\
\sum_{s^{\prime}} \frac{\mathrm{e}^{-\tilde{E}_{s^{\prime}}} \mathrm{e}^{\psi_{s^{\prime}}} \cos \phi_{s^{\prime}}}{\xi+\eta \mathrm{e}^{-\tilde{E}_{s^{\prime}} \mathrm{e}^{\psi_{s^{\prime}}} \cos \phi_{s^{\prime}}}}=N-1 .
\end{gathered}
$$

Equations from (10) to (12) completely solve the problem of the statistical description of the systems with any type of interaction. One or another state of the system is realized in accordance with the solution of the nonlinear equations. Among the solutions there are solutions which correspond to the spatial nonhomogeneous distribution of particles. So the Bose condensation is realized in the real space because we can say that an aggregation of particles is the Bose condensation of a sort. One of the possibilities of cluster formation was demonstrated in [2]; the nonhomogeneous distribution of particles which were characterized by the shielded Coulomb interaction for both the attraction and the repulsion just yielded stable clusters. Such type of the interaction was chosen owing to the existence of the inverse operator of the interaction energy in an explicit form. Nevertheless, it is a very difficult problem to find the inverse operator among the most widespread types of interactions. That is why, there is a necessity to develop methods which are capable of describing the particle distribution at any interaction. In $[4,5]$ the approach based on the selection of states in the form of a "combined variable" has been proposed. The combined variable has given us the chance to find connections between different fields (such as $\phi_{s}$ and $\psi_{s}$ in the present paper) along the extremal path that passes through the saddle-point.

\section{Combined variable and the selection of states}

Let us introduce the designation

$$
\Gamma_{s}=\frac{\mathrm{e}^{-\tilde{E}_{s}} \mathrm{e}^{\psi_{s}} \cos \phi_{s}}{\xi+\eta \mathrm{e}^{-\tilde{E}_{s}} \mathrm{e}^{\psi_{s}} \cos \phi_{s}} .
$$

The value $\Gamma_{s}$ includes the two field variables, $\psi_{s}$ and $\phi_{s}$, and the fugacity $\xi$. Inserting $\Gamma_{s}$ into the left hand side of equation (12) we will see that the total sum $\sum_{s} \Gamma_{s}$ is equal to the number of particles in the system studied

$$
\sum_{s} \Gamma_{s}=N-1
$$

This means that $\Gamma_{s}$ directly specifies the quantity of particles in the $s$-th state. Hence, the value $\Gamma_{s}$ might be considered as a characteristic of the number of particles 
contained in the $s$ th cluster. So, it is quite possible to consider $\Gamma_{s}$ to be the variable of particle number and, on the other hand, it may be called the combined variable owing to the fact that it combines the variables $\psi_{s}, \phi_{s}$, and $\xi$. Probably the introduction of the combined variable is a rough approximation, but it permits to determine the clusterization conditions in cases when the inverse operator of the interaction potential cannot be found.

Utilizing the variable (13) we can present the action (9) as a function of only two variables, $\Gamma_{s}$ and $\xi$, and it makes it possible to do away with the inverse operators $\tilde{v}_{s s^{\prime}}^{-1}$ and $\tilde{u}_{s s^{\prime}}^{-1}$ in the final expression.

If we multiply two sides of equation (11) by the function $\tilde{v}_{s s^{\prime \prime}}$ and then summing equation (11) over $s$, we obtain

$$
\psi_{s^{\prime}}=\sum_{s} \tilde{v}_{s s^{\prime}} \Gamma_{s}
$$

Now let us multiply the same equation (11) by $\psi_{s}$; then summing it over $s$ we acquire

$$
\sum_{s s^{\prime}} \tilde{v}_{s s^{\prime}}^{-1} \psi_{s^{\prime}} \psi_{s}=\sum_{s s^{\prime}} \tilde{v}_{s s^{\prime}} \Gamma_{s^{\prime}} \Gamma_{s}
$$

Multiplying equation (10) by $\tilde{u}_{s^{\prime \prime} s}$ and summing it over $s$ we have

$$
\sum_{s s^{\prime}} \tilde{u}_{s s^{\prime}}^{-1} \tilde{u}_{s^{\prime \prime} s} \phi_{s^{\prime}}=-\sum_{s} \tilde{u}_{s s^{\prime \prime}} \frac{\mathrm{e}^{-\tilde{E}_{s}} \mathrm{e}^{\psi_{s}} \sin \phi_{s}}{\xi+\eta \mathrm{e}^{-\tilde{E}_{s}} \mathrm{e}^{\psi_{s}} \cos \phi_{s}} .
$$

From relationship (13) one obtains

$$
\frac{1}{\xi} \mathrm{e}^{-\tilde{E}_{s}} \mathrm{e}^{\psi_{s}} \cos \phi_{s}=\frac{\Gamma_{s}}{1-\eta \Gamma_{s}}
$$

Let us substitute (18) into (17) taking into account equation (15) and using condition (4) for the left-hand side of equation (17). We gain

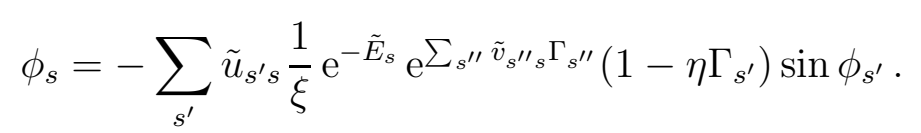

With the formula $\sin \phi_{s^{\prime}}=\sqrt{1-\cos ^{2} \phi_{s^{\prime}}}, \cos \phi_{s}$ can be substituted from (18) into (19). As a result, instead of (19) we have

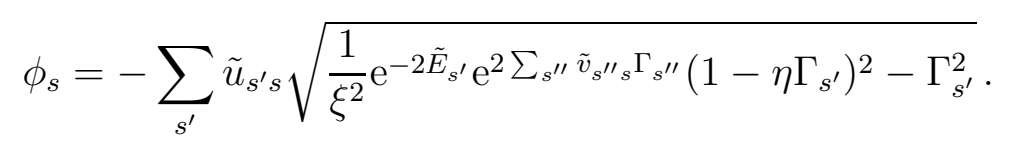

Consequently using equations (10) and (13) we get:

$$
\sum_{s s^{\prime}} \tilde{u}_{s s^{\prime}}^{-1} \phi_{s^{\prime}} \phi_{s}=-\sum_{s} \Gamma_{s} \phi_{s} \tan \phi_{s}
$$


or in the explicit form:

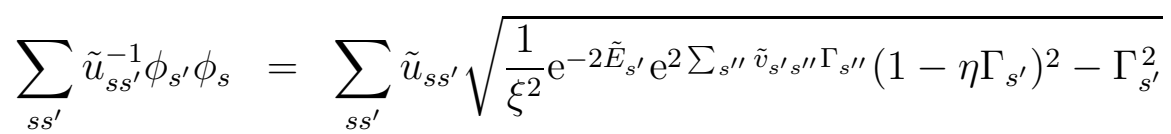

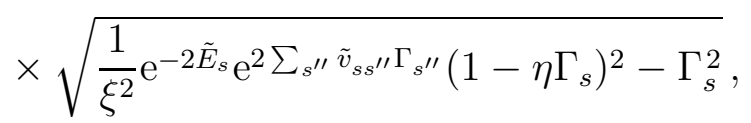

here, allowance is made for the negative sign of the field $\phi_{s}$ (see (19)).

Now using relationships (16) and (21) we can rewrite the action (9) in the point of extremum as follows:

$$
\begin{aligned}
& S=-\frac{1}{2} \sum_{s s^{\prime}} \tilde{v}_{s s^{\prime}} \Gamma_{s^{\prime}} \Gamma_{s}-\eta \sum_{s} \ln \left|1-\eta \Gamma_{s}\right|+(N-1) \ln \xi
\end{aligned}
$$

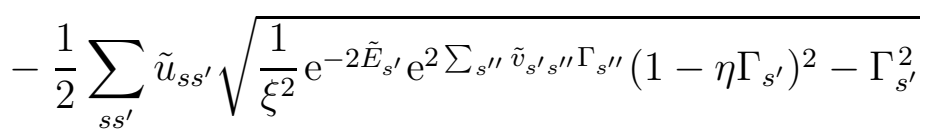

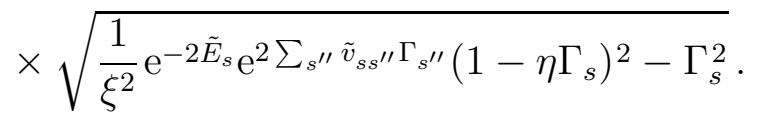

It has been shown in [5] that the minimum of the free energy based on the expression (22) is lower than that obtained in the framework of the mean field approximation. Indeed, let us assume following [7] that $N_{u}$ and $N_{v}$ are numbers of particles getting under the effect of the repulsive and attractive force, respectively. The number of particles $\Gamma_{s}$ in a cluster can be put constant equals $\Gamma$. Let the interaction energies in the range of the effect of these forces be changed to their average values. In this case expression (22) becomes:

$$
\begin{aligned}
S= & -\frac{1}{2}(N-1)\left\{N_{v} \Gamma \bar{U} \Gamma+N_{v} \Gamma \bar{V} \Gamma+2 \eta \ln |1-\eta \Gamma|\right\} \\
& -\frac{1}{2}(N-1)\left[N_{u} \bar{U}(1-\eta \Gamma) \mathrm{e}^{-2 \bar{E}} \mathrm{e}^{2 N_{v} \bar{V} \Gamma^{2}} \xi^{-2}-2 \ln \xi\right]
\end{aligned}
$$

where $\bar{U}$ and $\bar{V}$ are average values of the repulsive and attractive potential, respectively. Here, in the mean field approximation the expression in the curly brackets coincides with the free energy. The next term, i.e. the expression in the square brackets decreases the free energy at $\xi<1$. This inequality, $\xi<1$, corresponds to the coherent state of a system of interacting particles. Thus, the approach that is being developed can be considered as a general methodology of the mean field theory applying to the nonhomogeneous particle distribution.

Expression (22) is a function of only one variable $\Gamma$ and the fugacity $\xi$ and it is applicable to any kind of interaction, even though the inverse operator is unknown. The most beneficial advantage of expression (22) lies in the fact that it provides a way of finding the characteristics of the system such as its size, the number of particles in a cluster and the temperature of phase transition.

Now, let us pass to continual variables into the action (22). Inasmuch as we are interested in the nonhomogeneous distribution of particles in an indeterminate 
volume, let a radius $R$ be the fitting parameter of the system studied. Assume that the density of particles is distinguished from zero only in the cluster volume. Then equation (14) can be written as

$$
\Gamma K=N-1
$$

where $K$ is the number of clusters in the system and $\Gamma$ defined in expression (13) is the combined variable of the fields $\phi_{s}$ and $\psi_{s}$, and the fugacity $\xi$ in a cluster. As is seen from expression (24), the variable $\Gamma$ can be interpreted as the mean quantity of particles in a cluster. Then the passage to the continual presentation is realized by the substitution:

$$
\sum_{s} f_{s}=K \frac{1}{V} \int_{\text {cluster }} f(\vec{r}) \mathrm{d} \vec{r},
$$

here, the integration extends to the volume of a cluster and $V=\frac{4 \pi}{3} g^{3}$ is the effective volume occupied by one particle where $g$ is the distance between particles, i.e., the lattice constant.

Thus, in the continual presentation and with allowing for the assumption (14), we can transform the action (22) to the form:

$$
\begin{aligned}
S=- & \frac{1}{2 V^{2}} \int \mathrm{d} \vec{r} \int \mathrm{d} \vec{r}^{\prime} \tilde{v}\left(\vec{r}-\vec{r}^{\prime}\right) \Gamma\left(\vec{r}^{\prime}\right) \Gamma(\vec{r}) \\
- & \frac{1}{2 V^{2}} \int \mathrm{d} \vec{r} \int \mathrm{d} \vec{r}^{\prime} \tilde{u}\left(\vec{r}-\vec{r}^{\prime}\right) \Gamma\left(\vec{r}^{\prime}\right) \Gamma(\vec{r}) \\
& \times \sqrt{\mathrm{e}^{-2 \tilde{E}(\vec{r})} \mathrm{e}^{2 \int \tilde{v}\left(\vec{r}-\vec{r}^{\prime \prime}\right) \Gamma\left(\vec{r}^{\prime \prime}\right) \mathrm{d} \vec{r}^{\prime \prime}} \frac{1}{\xi^{2}}\left(\frac{1}{\Gamma(\vec{r})}-\eta\right)^{2}-1} \\
& \times \sqrt{\mathrm{e}^{-2 \tilde{E}(\vec{r})} \mathrm{e}^{2 \int \tilde{v}\left(\vec{r}-\vec{r}^{\prime \prime}\right) \Gamma\left(\vec{r}^{\prime \prime}\right) \mathrm{d} \vec{r}^{\prime \prime}} \frac{1}{\xi^{2}}\left(\frac{1}{\Gamma(\vec{r})}-\eta\right)^{2}-1} \\
+ & \eta \frac{1}{V} \int \ln |1-\eta \Gamma(\vec{r})| \mathrm{d} \vec{r}+(N-1) \ln \xi
\end{aligned}
$$

where the integrals are the same as in equation (25).

The integration is effected in accord with the rule:

$$
\begin{aligned}
\frac{1}{V} \int_{\text {cluster }} f(\vec{r}) \mathrm{d} \vec{r} & =\frac{1}{\frac{4 \pi}{3} g^{3}} \int_{0}^{2 \pi} \mathrm{d} \varphi \int_{0}^{\pi} \sin \theta \mathrm{d} \theta \int_{g}^{R} f(r) r^{2} \mathrm{~d} r \\
& =\frac{1}{\frac{4 \pi}{3} g^{3}} 4 \pi \int_{g}^{R} f(r) r^{2} \mathrm{~d} r
\end{aligned}
$$

Here we shift the limits of integration from 0 and $R$ to $g$ and $R$, respectively, as it allows one to eliminate the singularity of the integrand (the same procedure is made 
below in expression (29)). Thus, we can now normalize the integrals to the number of particles $\Gamma$ in a cluster, that is,

$$
\frac{1}{V} \int \mathrm{d} \vec{r}=\frac{1}{(4 \pi / 3) g^{3}} 4 \pi \int_{g}^{R} r^{2} \mathrm{~d} r=\frac{R^{3}-g^{3}}{g^{3}}=\Gamma-1 \cong \Gamma,
$$

the last approximation is, in fact, correct as we assume that $\Gamma$ satisfies the inequality $\Gamma \gg 1$.

Relation (28) allows us to introduce the dimensionless variable $x=r / g$ in the integral (27). Thereby, the rule of transformation from summation to integration, i.e. (25), becomes

$$
\frac{1}{K} \sum_{s} f_{s}=3 \int_{1}^{\Gamma^{1 / 3}} f(g x) x^{2} \mathrm{~d} x
$$

(once again, in expressions (27) we shift the limits of integration from 0 and $R$ to $g$ and $R$, respectively, as it allows one to eliminate the singularity of the integrand; the same shift is made in expression (29)).

Having integrated the action (26), we should exploit the following relationships

$$
\begin{aligned}
& \frac{1}{V^{2}} \int \mathrm{d} \vec{r} \int \mathrm{d} \vec{r}^{\prime} \tilde{v}\left(\vec{r}-\vec{r}^{\prime}\right) \Gamma(\vec{r}) \Gamma\left(\vec{r}^{\prime}\right)=\frac{1}{V^{2}} \int \mathrm{d} \vec{r} \tilde{v}(\vec{r}) \Gamma(\vec{r}) \cdot \int \mathrm{d} \vec{r}^{\prime} \Gamma\left(\vec{r}^{\prime}\right) \\
& \quad=\frac{1}{V^{2}} \int \mathrm{d} \vec{r} \tilde{v}(\vec{r}) \Gamma \cdot \Gamma \int \mathrm{d} \vec{r}^{\prime}=\frac{\Gamma^{2}}{V} \cdot \int \mathrm{d} \vec{r} \tilde{v}(\vec{r})=3 \Gamma^{2} \int_{1}^{\Gamma^{1 / 3}} \mathrm{~d} x x^{2} \tilde{v}(g x), \\
& \frac{1}{V^{2}} \int \mathrm{d} \vec{r} \int \mathrm{d} \vec{r}^{\prime \prime} \tilde{u}\left(\vec{r}-\vec{r}^{\prime \prime}\right) \Gamma(\vec{r}) \Gamma\left(\vec{r}^{\prime \prime}\right)=\frac{1}{V^{2}} \int \mathrm{d} \vec{r} \tilde{u}(\vec{r}) \Gamma^{2}=3 \Gamma^{2} \int_{1}^{\Gamma^{1 / 3}} \mathrm{~d} x x^{2} \tilde{u}(g x) .(31)
\end{aligned}
$$

With the transformations, we have used the step function: $\Gamma(r)=\Gamma \vartheta\left(\Gamma-r^{3} / g^{3}\right)$ where $\vartheta\left(\Gamma-r^{3} / g^{3}\right)=0$ if $r^{3} / g^{3} \geqslant \Gamma$ and $\vartheta\left(\Gamma-r^{3} / g^{3}\right)=1$ if $r^{3} / g^{3}<\Gamma$. Besides,

$$
\begin{aligned}
\frac{1}{V^{2}} \int \mathrm{d} \vec{r} \int \mathrm{d} \vec{r}^{\prime} \tilde{u}\left(\vec{r}, \vec{r}^{\prime}\right) f(\vec{r}) f\left(\vec{r}^{\prime}\right) & =\frac{1}{V^{2}} \int \mathrm{d} \vec{r} \tilde{u}(\vec{r}) f(\Gamma) \cdot \int \mathrm{d} \vec{r}^{\prime} f(\Gamma) \\
& =3 f^{2} \int_{1}^{\Gamma^{1 / 3}} \mathrm{~d} x x^{2} \tilde{u}(g x)
\end{aligned}
$$

where $f=\sqrt{\exp \left[-2 \tilde{E}+2 \Gamma \int \mathrm{d} \vec{r} \tilde{v}(\vec{r})\right]}$.

Now, if we introduce the designations

$$
a=3 \int_{1}^{\Gamma^{1 / 3}} \mathrm{~d} x x^{2} \tilde{u}(g x), \quad b=3 \int_{1}^{\Gamma^{1 / 3}} \mathrm{~d} x x^{2} \tilde{v}(g x),
$$


we will present the action (26) in the following final form

$$
S=K \cdot\left\{\frac{1}{2}(a-b) \Gamma^{2}-\frac{1}{2} \frac{1}{\xi^{2}}(1-\eta \Gamma)^{2} \mathrm{e}^{-2 \tilde{E}+2 b \Gamma}+\eta \ln |1-\eta \Gamma|\right\}+(N-1) \ln \xi .
$$

If we minimize the action (34) by the variable $\Gamma$, we will be able to define the number of particles in a cluster, the cluster size, and the temperature of phase transition that triggers the nonhomogeneous distribution of particles. It should be emphasized that the definition of the parameters of nonhomogeneous formations requires only explicit forms of potentials of interparticle interactions. Then, the system itself will select the realization which will provide for the minimum of the free energy. Nonetheless, first of all we assume the availability of clusters and only then the conditions and parameters of their existence are defined.

Studying the phase transition of clusters regarding the spatial homogeneous distribution of particles, we should take into account the behaviour of the fugacity $\xi=\mathrm{e}^{-\mu / k_{\mathrm{B}} T}$. Indeed, in the classical system the chemical potential:

$$
\mu=k_{\mathrm{B}} T \ln \left(\lambda_{T}^{3} n\right),
$$

where $n$ is the particle concentration and the de Broglie thermal wavelength of a particle with a mass $m$ :

$$
\lambda_{T}=h / \sqrt{3 m k_{\mathrm{B}} T} .
$$

Thus, in the classical case the fugacity

$$
\xi=\lambda_{T}^{3} n \ll 1 .
$$

In the case of a quantum system, the strong inequality does not hold; in this case $\xi<1$ and $\xi$ is only slightly smaller than the unit. Thereby, when we investigate the action (34) searching for clusters at the temperature $T<T_{\mathrm{c}}$, we may neglect the last term as $|\ln \xi|$ is rather small in comparison with the terms which include the highest orders of $\Gamma$. However, if we consider the action (34) scanning for the critical temperature $T=T_{\mathrm{c}}$, we should retain the last term because in this case $\xi$ tends to a very small magnitude such that $|\ln \xi| \gg 1$ and yet other terms decrease owing to the diminution of the value of $\Gamma$.

\section{Clusters in condensed media}

In this section we apply the methodology developed above to the systems of particles with long-range repulsion (attraction) and short range attraction (repulsion).

\subsection{Electrons on liquid helium surface}

The energy of electrostatic repulsion between electrons can be written as $u_{s s^{\prime}}=$ $1 / 4 \pi \epsilon_{0} \cdot Q^{2} /\left(r_{s}-r_{s^{\prime}}\right)$ where $Q$ is the effective charge of the electron in the helium film. The attraction between electrons caused by the deformation of the helium film can be taken as $v_{s s^{\prime}}=\frac{1}{2} \gamma_{\text {film }}\left(r_{s}-r_{s^{\prime}}\right)^{2}$ where the coefficient $\gamma_{\text {film }}$ characterizes 
the elasticity of the helium film in respect to the deformation caused by imbedded electrons (see, e.g. review [10]).

In this case the parameters $a$ and $b$ (33) of the action (34) are transformed to the following

$$
\begin{aligned}
& a=3 \frac{Q^{2} /\left(4 \pi \epsilon_{0} g\right)}{k_{\mathrm{B}} T} \int_{1}^{\Gamma^{1 / 3}} \frac{1}{x} x^{2} \mathrm{~d} x \cong \frac{3}{8 \pi \epsilon_{0}} \frac{Q^{2}}{g k_{\mathrm{B}} T} \Gamma^{2 / 3}, \\
& b=3 \frac{\gamma_{\text {film }} g^{2}}{2 k_{\mathrm{B}} T} \int_{1}^{\Gamma^{1 / 3}} x^{2} x^{2} \mathrm{~d} x \cong \frac{3}{10} \frac{\gamma_{\mathrm{film}} g^{2}}{k_{\mathrm{B}} T} \Gamma^{5 / 3}
\end{aligned}
$$

or

$$
a=\tilde{\alpha} \Gamma^{2 / 3}, \quad b=\tilde{\beta} \Gamma^{5 / 3}
$$

where

$$
\tilde{\alpha}=\frac{3}{8 \pi \epsilon_{0}} \frac{Q^{2}}{g k_{\mathrm{B}} T}, \quad \tilde{\beta}=\frac{3}{10} \frac{\gamma_{\mathrm{film}} g^{2}}{k_{\mathrm{B}} T}
$$

and $\alpha \gg \beta$ (hereafter the tilde over a symbol means the division by $k_{\mathrm{B}} T$ ). Since for electrons $\eta=+1$ and the inequality $\tilde{E} \equiv E / k_{\mathrm{B}} T \gg 1$ is legitimate (i.e., $\exp \left(-2 E / k_{\mathrm{B}} T\right) \ll 1$ where $E$ is the kinetic energy of an electron), the action (34) can be rewritten as

$$
S \cong K \cdot\left\{\frac{1}{2} \tilde{\alpha} \Gamma^{8 / 3}-\frac{1}{2} \tilde{\beta} \Gamma^{11 / 3}+\ln \Gamma+\Gamma \ln \xi\right\} .
$$

The extremum of the free energy is achieved at the solution of the equation $S^{\prime}(\Gamma)=$ 0 , or in the explicit form:

$$
\frac{4}{3} \tilde{\alpha} \Gamma^{5 / 3}-\frac{11}{6} \tilde{\beta} \Gamma^{8 / 3}+\frac{1}{\Gamma}+\ln \xi=0 .
$$

Retaining leading terms in equation (43), i.e. the highest powers to $\Gamma$, the equation is reduced to

$$
\frac{4}{3} \tilde{\alpha} \Gamma^{5 / 3}-\frac{11}{6} \tilde{\beta} \Gamma^{8 / 3} \approx 0
$$

and hence the solution to it is equal to

$$
\Gamma=\frac{8}{11} \frac{\alpha}{\beta} \equiv \frac{40}{33} \frac{n Q^{2}}{\epsilon_{0} \gamma_{\text {film }}}
$$

where $n=\left(\frac{4 \pi}{3} g^{3}\right)^{-1}$ is the concentration of electrons in a cluster. The sufficiency is also satisfied, i.e., $S^{\prime \prime}(\Gamma)>0$ with the solution (45) and, therefore, it actually determines the minimum of the action (42).

The critical temperature $T_{\mathrm{c}}$ is defined from the condition $\Gamma=2$, i.e. when the temperature is so high that no more than two particles are capable of interconnecting. In this case equation (43) turns into

$$
k_{\mathrm{B}} T_{\mathrm{c}}=\frac{-4 \alpha \cdot 2^{8 / 3}+\frac{11}{2} \beta \cdot 2^{11 / 3}}{3 \ln \xi} \approx-\frac{12}{\pi}\left(\frac{2 \pi}{3}\right)^{1 / 3} \frac{Q^{2} n^{1 / 3}}{\epsilon_{0} \ln \xi} .
$$


In the first approximation we may allow that in the critical point the fugacity $\xi \equiv$ $\mathrm{e}^{-\mu / k_{\mathrm{B}} T}$ reaches its classical meaning $\lambda_{T}^{3} n$ (see expressions (35) and (36)). Expanding function $\ln \xi$ in a Taylor series, we obtain $\ln \lambda_{T}^{3} n \approx 3\left(\lambda_{T} n^{1 / 3}-1\right)$. Substituting the expansion in equation (46) and taking into account expression (36) for $\lambda_{T}$, we arrive at the following equation for $T_{\mathrm{c}}$

$$
k_{\mathrm{B}} T_{\mathrm{c}} \approx \frac{4}{\pi}\left(\frac{2 \pi}{3}\right)^{1 / 3} \frac{\mathrm{e}^{2} n^{1 / 3}}{\epsilon_{0}}
$$

Now, let us assign the concrete numerical values to the parameters. Let the charge $Q$ be the elementary charge $e$. Put $n=2.4 \cdot 10^{19} \mathrm{~m}^{-3}$ and $\gamma_{\text {film }}=8.4 \cdot 10^{-14} \mathrm{~N} / \mathrm{m}$. Then, setting these values into expression (45), we get the number of electrons in a cluster: $\Gamma \approx 10^{8}$. For the radius of the cluster we have $R=(3 \Gamma / 4 \pi n)^{1 / 3} \approx 10^{-4} \mathrm{~m}$. The critical temperature $T_{\mathrm{c}}$ estimated from expression (47) is of the order of $100 \mathrm{~K}$ that is far beyond the critical temperature $(4 \mathrm{~K})$ of the liquid helium film. Thus, the thermodynamic condition of the liquid helium stability sets limits to the real temperature $T_{\mathrm{c}}$ of the cluster existence.

The behaviour of electrons in the liquid helium film has been studied by many researchers [10]. An experimental appraisal of the mean number of electrons in a near-surface bubblon was approximately equal to $10^{8}$. The radius of the bubblon was estimated as $10^{-2} \mathrm{~cm}$. In such a manner our qualitative evaluation of the values $\Gamma$ and $R$ is in agreement with the experimental data.

\subsection{Shielding Coulomb potential}

Let particles be repulsed by the shielding Coulomb potential $u_{s s^{\prime}}=1 / 4 \pi \epsilon_{0} \times$ $Q^{2} \mathrm{e}^{-\kappa\left|r_{s}-r_{s^{\prime}}\right|} \times\left(r_{s}-r_{s^{\prime}}\right)^{-1}$, where $\kappa$ is the effective radius of screening of nucleons in a nucleus, and attracted by the potential $v_{s s^{\prime}}=\frac{1}{2} \gamma\left(r_{s}-r_{s^{\prime}}\right)^{2}$ that can be created for an external reason (so $\gamma$ is the force constant of an off-site elastic field). In this case, the parameters $a$ and $b$ (33) of the action (34) become:

$$
\begin{aligned}
a & =3 \frac{Q^{2}}{4 \pi \epsilon_{0} g k_{\mathrm{B}} T} \int_{1}^{\Gamma^{1 / 3}} \frac{1}{x} \mathrm{e}^{-\kappa g x} x^{2} \mathrm{~d} x \\
& =\frac{3 Q^{2}}{4 \pi \epsilon_{0} \kappa^{2} g^{3} k_{\mathrm{B}} T}\left[-\left(\kappa g \Gamma^{1 / 3}+1\right) \mathrm{e}^{-\kappa g \Gamma^{1 / 3}}+(\kappa g+1) \mathrm{e}^{-\kappa g}\right] .
\end{aligned}
$$

We will consider weight nuclei $(\Gamma \gg 1)$ and, therefore, $\Gamma^{1 / 3}$ should be several times as large as the unit. Therefore, at the first approximation we may neglect the terms with $\mathrm{e}^{-\kappa g \Gamma^{1 / 3}}$ and then expression (48) becomes:

$$
a \simeq \frac{3}{4 \pi \epsilon_{0}} \frac{Q^{2}}{\kappa^{2} g^{3} k_{\mathrm{B}} T}(\kappa g+1) \mathrm{e}^{-\kappa g} .
$$

Now

$$
b=3 \frac{\gamma g^{2}}{2 k_{\mathrm{B}} T} \int_{1}^{\Gamma^{1 / 3}} x^{2} x^{2} \mathrm{~d} x \cong \frac{3}{10} \frac{\gamma g^{2}}{k_{\mathrm{B}} T} \Gamma^{5 / 3}
$$


Let us rewrite these expressions in the form:

$$
a=\tilde{\alpha}, \quad b=\tilde{\beta} \Gamma^{5 / 3},
$$

where

$$
\tilde{\alpha}=\frac{3}{4 \pi \epsilon_{0}} \frac{Q^{2}}{\kappa^{2} g^{3} k_{\mathrm{B}} T}(\kappa g+1) \mathrm{e}^{-\kappa g}, \quad \tilde{\beta}=\frac{3}{10} \frac{\gamma g^{2}}{k_{\mathrm{B}} T}
$$

and $\alpha \gg \beta$. Let us now substitute expressions (49) and (50) for $a$ and $b$ in the action (34). With regard to inequality $\tilde{E} \gg 1$ and with allowance for $\eta=+1$ we will get:

$$
S \cong K \cdot\left\{\frac{1}{2} \tilde{\alpha} \Gamma^{2}-\frac{1}{2} \tilde{\beta} \Gamma^{11 / 3}+\ln \Gamma+\Gamma \ln \xi\right\} .
$$

The extremum of the action $S$ is reached with resolving the equation $S^{\prime}(\Gamma)=0$, or explicitly

$$
\tilde{\alpha} \Gamma-\frac{11}{3} \tilde{\beta} \Gamma^{8 / 3}+\frac{1}{\Gamma}+\ln \xi=0 .
$$

Retaining two highest order terms in equation (54), we obtain:

$$
\begin{aligned}
\Gamma & \approx\left(\frac{6}{11} \frac{\alpha}{\beta}\right)^{3 / 5} \\
& =\frac{4}{3} \pi^{2 / 5} n\left\{\frac{15}{11} \frac{Q^{2}}{\epsilon_{0} \kappa^{2} \gamma}\left[\left(\frac{3}{4 \pi}\right)^{1 / 3} \frac{\kappa}{n^{1 / 3}}+1\right]\right\}^{3 / 5} \exp \left[-\frac{3}{5}\left(\frac{3}{4 \pi}\right)^{1 / 3} \frac{\kappa}{n^{1 / 3}}\right]
\end{aligned}
$$

The critical temperature $T_{\mathrm{c}}$ of the cluster destruction is defined from equation (54) if one puts $\Gamma=2$ :

$$
k_{\mathrm{B}} T_{\mathrm{c}} \approx \frac{4 \alpha}{-\ln \xi}
$$

However, having found the fugacity $\xi$, we now cannot follow the procedure described in the previous subsection. In a nucleus, the temperature and the thermal de Broglie wavelength are different. In other words, we should exploit the laws of high energy physics, i.e. that the total energy of a nucleon is linked with the momentum of the nucleon by the relation $E=\sqrt{p^{2} c^{2}+m^{2} c^{4}}$. Hence, in this case

$$
\frac{3}{2} k_{\mathrm{B}} T=c \sqrt{p^{2}+m^{2} c^{2}}
$$

and, therefore,

$$
p \simeq 3 k_{\mathrm{B}} T / 2 c .
$$

Thus, the de Broglie thermal wavelength of the nucleon in a nucleus is determined as

$$
\lambda_{T}=\frac{2 h}{3 c k_{\mathrm{B}} T} .
$$

Expanding function $\ln \xi=\ln \left(\lambda_{T}^{3} n\right)$ in equation (56) in a Taylor series we obtain $\ln \xi \simeq-3$ and, therefore, equation (53) becomes

$$
k_{\mathrm{B}} T_{\mathrm{c}} \approx \frac{4}{3} \alpha \equiv \frac{4}{3} \frac{n Q^{2}}{\epsilon_{0} \kappa^{2}}\left\{\left(\frac{3}{4 \pi}\right)^{1 / 3} \frac{\kappa}{n^{1 / 3}}+1\right\} \exp \left[-\left(\frac{3}{4 \pi}\right)^{1 / 3} \frac{\kappa}{n^{1 / 3}}\right] .
$$


The results obtained in this section may account for the reasons of the atomic nucleus stability from the microscopic viewpoint. The shielding Coulomb potential $u=1 / 4 \pi \epsilon_{0} \cdot Q^{2} \mathrm{e}^{-\kappa r} / r$ is the typical nuclear (Yukawa) potential that provides for repulsion between protons. The potential $v=\frac{1}{2} \gamma r^{2}$, which is applied to nucleons, ensures their mutual attraction. For example, setting $\gamma=4 \cdot 10^{17} \mathrm{~N} / \mathrm{m}$, we get from expression $(55) \Gamma \approx 30$, which corresponds to the number of nucleons in a nucleus of zinc. The critical temperature of fission of a proton-neutron pair calculated from expression $(60)$ results in $T_{\mathrm{c}} \sim 10^{11} \mathrm{~K}$. Note that this value is several times of magnitude greater than the typical temperature of a weight nucleus (the nucleus temperature is of the order of the Coulomb repulsion between protons in the nucleus).

\subsection{Gravitating masses with Hubble expansion}

Gravitational physics may also be assigned to the condensed matter [11]. The gravitational attraction between particles, i.e. big masses, is beyond question. However, the uniform character of the Hubble expansion is doubtful. We will use only the fact that an additional kinetic energy of particles $E_{s s^{\prime}}=\frac{1}{2} m\left(w_{s}-w_{s^{\prime}}\right)^{2}$ is associated with such an expansion. The relative velocity $w_{s}-w_{s^{\prime}}$ of particles which are found in points $s$ and $s^{\prime}$, respectively, pertains to the relative distance between the particles, since

$$
w_{s}-w_{s^{\prime}}=H\left(r_{s}-r_{s^{\prime}}\right),
$$

where $H$ is the Hubble constant. In this connection, we will consider a model system of identical gravitating masses, i.e. stars, with the attraction $v_{s s^{\prime}}=G m^{2} /\left(r_{s}-r_{s^{\prime}}\right)$ and the effective repulsion $u_{s s^{\prime}}=\frac{1}{2} H^{2} m\left(r_{s}-r_{s^{\prime}}\right)^{2}$. For such kinds of interactions one has for the parameters $a$ and $b(33)$ :

$$
\begin{aligned}
& a=3 \frac{g^{2} H^{2} m}{2 k_{\mathrm{B}} T} \int_{1}^{\Gamma^{1 / 3}} x^{2} x^{2} \mathrm{~d} x \cong \frac{3}{10} \frac{g^{2} H^{2} m}{k_{\mathrm{B}} T} \Gamma^{5 / 3}, \\
& b=3 \frac{G m^{2}}{g k_{\mathrm{B}} T} \int_{1}^{\Gamma^{1 / 3}} \frac{1}{x} x^{2} \mathrm{~d} x \cong \frac{3}{2} \frac{G m^{2}}{g k_{\mathrm{B}} T} \Gamma^{2 / 3}
\end{aligned}
$$

or

$$
a=\tilde{\alpha} \Gamma^{5 / 3}, \quad b=\tilde{\beta} \Gamma^{2 / 3},
$$

where

$$
\tilde{\alpha}=\frac{3}{10} \frac{g^{2} H^{2} m}{k_{\mathrm{B}} T}, \quad \tilde{\beta}=\frac{3}{2} \frac{G m^{2}}{g k_{\mathrm{B}} T} .
$$

Inasmuch as gravitating masses are associated with the Bose statistics, i.e. $\eta=-1$, the action (34) is rewritten as follows

$$
S \simeq K \cdot\left\{\frac{1}{2} \tilde{\alpha} \Gamma^{11 / 3}-\frac{1}{2} \tilde{\beta} \Gamma^{8 / 3}-\ln \Gamma+\Gamma \ln \xi\right\} .
$$


In expression (65) we omit the exponent term because of the assumption that the temperature $T$ of the universe is very close to the absolute zero (at small $T$ the exponent in expression (34) tends to zero). Then the equation $S^{\prime}(\Gamma)=0$ becomes:

$$
\frac{11}{6} \tilde{\alpha} \Gamma^{8 / 3}-\frac{4}{3} \tilde{\beta} \Gamma^{5 / 3}-\frac{1}{\Gamma}+\ln \xi=0 .
$$

Here, $\alpha \ll \beta$. Retaining the highest order terms in equation (66) we immediately obtain the solution

$$
\Gamma \simeq \frac{8}{11} \frac{\beta}{\alpha}=\frac{120 \pi}{33} \frac{G m n}{H^{2}} .
$$

where $n=\left(\frac{4 \pi}{3} g^{3}\right)^{-1}$ is the concentration of particles, i.e. stars.

The equation for the critical temperature $T_{\mathrm{c}}$ that determines the break-up of two coupled masses is obtained from equation (66) in which we set $\Gamma=2$ (and the exponent term is neglected). With the inequality $\alpha \ll \beta$, we get

$$
k_{\mathrm{B}} T_{\mathrm{c}} \approx \frac{2^{2 / 3}}{3} \frac{\beta}{\ln \xi} \equiv\left(\frac{2 \pi}{3}\right)^{1 / 3} \frac{G m^{2} n^{1 / 3}}{\ln \xi} .
$$

By definition, the fugacity $\xi=\mathrm{e}^{-\mu / k_{\mathrm{B}} T}$. At the transition from the Bose statistics to the classical one, the chemical potential gradually decreases with temperature starting from a value $\mu \sim 0$ (but still $\mu<0$ ) to $\mu \ll 0$. So, the function $\ln \xi$ in equation (68) is strongly negative: $\ln \xi=-|\mu| / k_{\mathrm{B}} T$. This signifies that the critical temperature in the system of gravitating masses is absent. In other words, in the universe, masses tend to aggregation at any conditions.

Thus, the results obtained demonstrate that the availability of the Hubble expansion is the decisive factor in the formation of galaxies in the universe (note that without $H$, masses in the universe will merely tend to the total mergence and any spatial distribution will not be observed). Actually, expression (67) shows that Hubble's constant, $H \approx 50 \mathrm{~km} /(\mathrm{s} \cdot \mathrm{Mpsc})=1.6 \cdot 10^{-18} \mathrm{~s}^{-1}$, is one of the main parameters that determines the steady state of masses in a cluster, i.e., galaxy. So the greater the mean mass $m$, the greater the number $\Gamma$ of stars in a galaxy.

\section{Conclusion}

In the present paper the statistical description of a system of interacting particles, which enables the study of their spatial nonhomogeneous distribution, has been proposed. One of the most important properties of our model is the presentation of the total potential of interacting particles in the form of the paired energy of attraction and repulsion. We have started from the Hamiltonian that describes interacting particles located in the knots of the three-dimensional lattice and applied the quantum field theory methodology based on the functional integrals for the description of the partition function of the systems studied.

We have shown that the solutions of equations characterizing the behaviour of the field variables yield a complete resolving of the initial statistical task. The solutions 
may correspond to both the homogeneous and nonhomogeneous distributions of particles and only the character of interparticle interaction and the temperature define the type of the solution.

The free energy represented through field variables in the saddle-point has allowed us to investigate special conditions, which provide for the formation of clusters in a system with the initially homogeneous particle distribution. The conditions are also responsible for the size of clusters and for the temperature of phase transition to the cluster state.

The approach proposed does not need fitting parameters: the cluster size and the temperature of phase transition to the cluster state are completely determined by the value and character of paired potentials in the systems of interacting particles. Besides, the introduction of a peculiar type of the combined variable has simplified the investigation procedure. Specifically, the procedure has provided a way of obtaining the necessity criterion of the transition of a system of separate particles into the spatial nonhomogeneous state.

The authors are very thankful to the referees for the concrete constructive remarks that made significant improvements of the contents of the paper possible.

\section{References}

1. Kleinert H. Gauge Fields in Condensed Matter. Singapore, World Scientific, 1989.

2. Belotsky E.D., Lev B.I. Formation of clusters in condensed media. // Theor. Math. Phys., 1984, vol. 60, No. 1, p. 120-132 (in Russian).

3. Lev B.I., Zhugaevich A.Ya. Statistical description of model systems of interacting particles and phase transitions accompanied by cluster formation. // Phys. Rev. E, 1998, vol. 57, No. 6, p. 6460-6469.

4. Krasnoholovets V., Lev B.I. On mechanism of H-associate polymerazation in alkyland alkoxybenzene acids. // Ukrain. Fiz. Zhurn., 1994, vol. 33, No. 3, p. 296-300 (in Ukrainian).

5. Belotsky E.D. Nonlinear and nonequilibrium spartial-limited structures (questions of theory). Thesis, Inst. of Phys., Kyiv, 1992, 242 p. (in Ukrainian).

6. Khachaturian A.G. The Theory of Phase Transitions and the Structure of Solid Mixtures. Moscow, Nauka, 1974 (in Russian).

7. Magalinsky V.B. Thermodynamics of a one-dimensional model of particles with a Coulomb interaction. // JETP, 1965, vol. 48, No. 1, p. 167-174 (in Russian).

8. Edwards S.F. The statistical thermodynamics of a gas with long and short-range forces. // Phil. Mag., 1959, vol. 4, No. 46, p. 1171-1182.

9. Edwards S.F., Lennard A.J. Exact statistical mechanics of a one dimensional system with Coulomb forces. II. The method of functional integration. // J. Math. Phys., 1962, vol. 3, No. 4, p. 778-792.

10. Edelman P.S. Levitating electrons. // Uspekhi Fiz. Nauk, 1980, vol. 130, No. 4, p. 675706 (in Russian).

11. Saslaw W.C. Gravitational Physics of Stellar and Galactic Systems. Cambridge, N.Y., Melbourne, Sydney, Cambridge University Press, 1989. 


\title{
Системи частинок з взаємодією і утворення кластерів у конденсованому середовищі
}

\author{
В.Красноголовець, Б.Лев
}

Інститут фізики НАН України, 03028 Київ, проспект Науки, 46

Отримано 21 грудня 2000 р., в остаточному вигляді 29 грудня 2002 р.

Досліджено поведінку системи частинок з різними типами взаємодії. Пропонований підхід дозволяє описати системи взаємодіючих частинок статистичними методами, беручи до уваги просторову неоднорідність розподілу частинок, тобто, формування кластерів. Для кластерів оцінено: їхній розмір, число частинок в кластері і температуру фазового переходу до стану кластера. Розглянуто три системи: електрони на поверхні рідкого гелію; частинки, що перебувають в пружньому полі і додатково взаємодіють за допомогою екранованого кулонівського потенціалу (наприклад, нуклони в ядрі); частинки 3 гравітаційним потенціалом при наявності Хаблівського розширення.

Ключові слова: статистична механіка, міжчастинкова взаємодія, кластери

PACS: $34.10 .+x, 36.90 . f$ 
